\title{
CALIBRAÇÃO DE MÉTODOS DE EXTRAÇÃO DE POTÁSSIO EM SOLOS CULTIVADOS SOB SISTEMA PLANTIO DIRETO(1)
}

\author{
Jairo André Schlindwein ${ }^{(2)}$, Leandro Bortolon ${ }^{(3)} \&$ Clesio Gianello ${ }^{(4)}$
}

\begin{abstract}
RESUMO
As recomendações de adubação no Rio Grande do Sul e em Santa Catarina são baseadas nos estudos realizados em culturas cultivadas no sistema convencional de preparo do solo e nos conhecimentos acumulados ao longo dos anos. Nesse período, ocorreram mudanças no sistema de cultivo, na profundidade de amostragem do solo e no aumento de rendimento das culturas. Isso pode alterar os teores críticos de $\mathrm{K}$, as faixas de fertilidade e as quantidades de fertilizante para as culturas. Este trabalho teve por objetivo calibrar os teores de K disponível, extraído pelos métodos Mehlich-1, Mehlich-3 e resina de troca iônica, para as culturas de soja, trigo e milho cultivadas no sistema plantio direto. Para isso, utilizaram-se amostras de terra de 17 experimentos e resultados de rendimento de grãos de 48 avaliações das culturas de soja, trigo e milho cultivadas em sistema plantio direto, com doses de K, no Rio Grande do Sul; a partir das funções de produção, determinaram-se a curva de calibração e as faixas de fertilidade. Os resultados indicaram que as respostas das plantas à adubação com $\mathrm{K}$ foram maiores, nesta sequência: milho, trigo e soja; os teores críticos de K com a solução de Mehlich-1, Mehlich-3 e resina foram de 89,5; 91,6; e 83,8 $\mathrm{mg} \mathrm{kg}^{-1}$ de $\mathrm{K}$ na camada de 0-20 cm de profundidade e de 117,$2 ; 116,1$; e $102,5 \mathrm{mg} \mathrm{kg}^{-1}$ de $\mathrm{K}$ na camada de $0-10 \mathrm{~cm}$ de profundidade, respectivamente, e são maiores do que os recomendados pela CFSRS/ SC (2004) para as culturas soja, trigo e milho cultivadas no sistema plantio direto.
\end{abstract}

Termos de indexação: faixas de fertilidade, teor crítico de potássio, adubação potássica, métodos de análise de solo.

\footnotetext{
(1) Trabalho financiado pelo Laboratório de Solos da Universidade Federal do Rio Grande do Sul - UFRGS. Recebido para publicação em 6 de maio de 2009 e aprovado em 11 de agosto de 2010.

(2) Professor Adjunto da Universidade Federal de Rondônia - UNIR. Av. Norte Sul, 7300, CEP 76940-000 Rolim de Moura (RO). E-mail: jairojas@unir.br

(3) Pesquisador da Embrapa Pesca e Aquicultura. Qd 103 Sul, I, Avenida JK 164, CEP 77015-012 Palmas (TO). E-mail: leandro.bortolon@embrapa.br

(4) Professor Associado do Departamento de Solos, Universidade Federal do Rio Grande do Sul - UFRGS. Av. Bento Gonçalves 7712, CEP 91540-000 Porto Alegre (RS). E-mail: cgianello@hotmail.com.br
} 


\title{
SUMMARY: CALIBRATION FOR POTASSIUM EXTRACTION METHODS FOR NO-TILLAGE SOILS
}

\begin{abstract}
Fertilizer recommendations in Rio Grande do Sul and Santa Catarina (RS and SC) states are based on studies of crops grown under conventional tillage and knowledge accumulated over the years. In this period, the crop system and soil sampling depth were altered and crop yields increased; this can affect the critical values of $K$, the fertility status and fertilizer quantity required by crops. This study aimed to determine the levels of available $K$ extracted by Mehlich-1, Mehlich-3 and ion-exchange resin from no tillage soils under soybean, wheat and corn. For this purpose, the K levels of soil samples from 17 experiments were used as well as grain yield data of soybean, wheat and corn grown under no-tillage in 48 studies carried out in RS. Based on the production functions the calibration curve and fertility status were determined. Results indicated that the plant response to K fertilization followed the order: corn $>$ wheat $>$ soybean. The critical K values extracted with Mehlich-1, Mehlich-3 and resin were 89.5, 91; 6 and $83.8 \mathrm{mg} \mathrm{kg}^{-1} \mathrm{~K}$ in the 0-20 $\mathrm{cm}$ layer and 117.2; 116.1 and $102.5 \mathrm{mg} \mathrm{kg}^{-1} \mathrm{~K}$ in the 0-10 cm layer, respectively, and exceeded those recommended by CFSRS / SC (2004) for soybean, wheat and corn grown under no-tillage.
\end{abstract}

Index terms: soil fertility status, critical soil $K$; K fertilization; soil analysis method.

\section{INTRODUÇÃO}

Calibrar um método de análise do solo consiste em relacionar o teor de um nutriente disponível no solo utilizando-se para tal um método de avaliação com respostas de plantas cultivadas no campo (índice de crescimento, teor de um nutriente e, ou, rendimento de cultura) (Rouse, 1968; Cate \& Nelson, 1973; Anghinoni \& Volkweiss, 1984; Evans, 1987).

As recomendações de adubação e calagem no Rio Grande do Sul e Santa Catarina (CFSRS/SC, 1995, 2004) são baseadas nos estudos de calibração de $P, K$ e N, feitos no final da década de 1960 a meados da década de 1980 e nos conhecimentos acumulados até as edições das recomendações. Há dúvidas quanto à atualização dos valores das tabelas, pois ocorreram mudanças nos cultivos nos últimos anos, destacandose: (a) a maior parte das áreas de produção com soja, trigo e milho cultivadas no Rio Grande do Sul é sob o sistema plantio direto, que promove a concentração superficial e subsuperficial de $\mathrm{P}, \mathrm{K}$, outros nutrientes e matéria orgânica, decorrentes das adubações e da reciclagem de nutrientes (Eltz et al., 1989; Schlindwein \& Anghinoni, 2000), e, assim, amostras de solo retiradas na camada de $0-10 \mathrm{~cm}$ devem resultar em maior valor de $\mathrm{K}$ em relação à camada de $0-20 \mathrm{~cm}$, sem que necessariamente aumente o rendimento das culturas (Schlindwein \& Anghinoni, 2000); (b) o rendimento médio das principais culturas cultivadas em campo aumentou na década de 1970, com média dos dados disponíveis da década de 2000 no Rio Grande do Sul em torno de 48, 116 e $125 \%$ para soja, trigo e milho, respectivamente (Emater/RS, 1998; CONAB, 2009). Esses aumentos podem ser devidos à utilização de variedades mais produtivas e, ou, de técnicas mais avançadas de produção. Entretanto, a exigência de nutrientes também aumentou com a maior exportação de nutrientes. Com isso, além do maior teor crítico, deve ser necessária uma dose maior de fertilizante.

Os primeiros estudos de calibração no Rio Grande do Sul foram realizados sobre a cultura do trigo em 16 locais, com a participação de diversas instituições. Esses resultados, somados aos dados de outras culturas, deram origem às "Recomendações de adubação e de calcário para as principais culturas do Estado do Rio Grande do Sul” (Mielniczuk et al., 1969). Nas tabelas de recomendações de adubação publicadas até 1987, o nível crítico de K na camada de solo de 0 $20 \mathrm{~cm}$ era de $60 \mathrm{mg} \mathrm{dm}^{-3}$ (Mielniczuk et al., 1969; Universidade..., 1973; Tabelas..., 1976; Manual..., 1981). O nível crítico de K é um valor estimado, em que, com teores de $\mathrm{K}$ disponíveis no solo acima desse valor, as plantas apresentam baixa ou nula probabilidade de resposta à adubação potássica, e com teores disponíveis de $\mathrm{K}$ no solo abaixo desse valor as plantas dão resposta a essa adubação inversamente proporcional aos teores de K disponíveis no solo (Cate \& Nelson, 1973). A partir de 1987, o nível crítico de K passou a $80 \mathrm{mg} \mathrm{dm}^{-3}$ (Siqueira et al., 1987; CFSRS/ SC, 1995; 2004). Em 2004, esse nível foi ajustado para diferentes CTCs, sendo de 45, 60 e $90 \mathrm{mg} \mathrm{dm}^{-3}$ na camada de $0-20 \mathrm{~cm}$ de profundidade no solo cultivado no preparo convencional, ou na camada de $0-10 \mathrm{~cm}$ no plantio direto para CTC $<5 ; 5-15 \mathrm{e}>15 \mathrm{cmol}_{\mathrm{c}} \mathrm{dm}^{-3}$, respectivamente (CFSRS/SC, 2004).

Outros estudos de calibração de $\mathrm{K}$ realizados na Região Sul do Brasil, como os de Borkert et al. (1993) no Paraná e Scherer (1998) em Santa Catarina, encontraram nível crítico de K de 40 e de $63 \mathrm{mg} \mathrm{dm}^{-3}$, respectivamente, na camada de $0-20 \mathrm{~cm}$, em estudos realizados por vários anos com a cultura da soja cultivada no preparo convencional em apenas um 
latossolo cada. Recentemente, Brunetto et al. (2005), em dois argissolos do Rio Grande do Sul cultivados com soja e milho; e Wendling et al. (2008), em quatro latossolos e três argissolos no Paraguai, encontraram nível crítico de 42 e de $75 \mathrm{mg} \mathrm{dm}^{-3}$, respectivamente, na camada de $0-10 \mathrm{~cm}$ de profundidade de solos cultivados no sistema plantio direto.

O nível crítico de $\mathrm{K}$ na camada de $0-20 \mathrm{~cm}$ de profundidade, determinado por Mehlich-1, no Programa de Recomendação de Fertilizantes em Minas Gerais é de $70 \mathrm{mg} \mathrm{dm}^{-3}$ (CFSEMG, 1999) e no Cerrado brasileiro, de 40 e $80 \mathrm{mg} \mathrm{dm}^{-3}$, em solos com $\left.\mathrm{CTC}_{(\mathrm{pH}} 7,0\right)$ menor do que 4 e superior ou igual a $4 \mathrm{cmol}_{\mathrm{c}} \mathrm{dm}^{-3}$, respectivamente (Sousa \& Lobato, 2002). No Estado de São Paulo, o teor de K considerado médio é de 1,5 a 3,0 $\mathrm{mmol}_{\mathrm{c}} \mathrm{dm}^{-3}$, determinado pelo método da resina (Raij et al., 1997). Em Arkansas, EUA, o nível crítico de $\mathrm{K}$ na camada de $0-10 \mathrm{~cm}$, determinado por Mehlich-3 em 32 locais e anos de cultivos de arroz, foi de $80 \mathrm{mg} \mathrm{kg}^{-1}$ (Slaton et al., 2009).

O nível crítico de K é diferente nos diversos solos devido aos mecanismos de determinação do nutriente pelos métodos testados e às respostas das plantas à adubação com K, influenciadas pelas combinações dos fatores de produção. No Rio Grande do Sul e em Santa Catarina, o nível crítico de K determinado por Mehlich1 foi modificado ao longo dos anos, em razão dos estudos realizados e da evolução dos conhecimentos, entretanto poucos trabalhos foram feitos com cultivos em sistema plantio direto, com anos de avaliações e em diferentes locais. Assim, este trabalho teve por objetivos calibrar (relacionar) os teores do nutriente $\mathrm{K}$ no solo, determinado pelos métodos Mehlich-1, Mehlich-3 e resina de troca iônica, com o rendimento das culturas de soja, trigo e milho cultivadas sob sistema plantio direto em diferentes locais e anos de cultivo; e, a partir da determinação do teor crítico de K, estabelecer as faixas de fertilidade desse nutriente no solo.

\section{MATERIAL E MÉTODOS}

Os experimentos utilizados neste estudo foram conduzidos por diferentes instituições de ensino, pesquisa e cooperativas no Rio Grande do Sul, Brasil, e em vários tipos de solo. Os experimentos apresentavam diferentes épocas de cultivo, tempo de condução, delineamentos experimentais, tratamentos e objetivos da própria instituição. Foram utilizados os experimentos em que foi possível obter as amostras de terra dos tratamentos com doses crescentes de $\mathrm{Ke}$ os respectivos dados de produção das culturas cultivados sob sistema plantio direto. No quadro 1 são apresentadas as culturas avaliadas e os solos com alguns atributos.

Um experimento foi instalado na Estação Experimental Agronômica da Universidade Federal do Rio Grande do Sul em 2000, com cultivo de milho sob sistema plantio direto nas doses de $\mathrm{K}(0,12,23$, 46 e $93 \mathrm{~kg} \mathrm{ha}^{-1}$ de $\mathrm{K}_{2} \mathrm{O}$ ) distribuídas a lanço na superfície do solo. Em 2001 foi feita a reaplicação dessas mesmas doses, na superfície, em cultivo de trigo.

Os experimentos nas cooperativas (COTRISOJA, COTRIJAL, COTREL, COPALMA, COTRIJUI, COTRIPAL) em convênio com a FUNDACEP apresentaram o mesmo esquema fatorial (4 x 4), composto por culturas e doses de K aplicadas sempre

Quadro 1. Culturas, tipo de solo, atributos físico e de fertilidade de solo no tratamento sem adição de potássio dos experimentos com doses de $\mathrm{K}_{2} \mathrm{O}$, realizados por diferentes instituições no Rio Grande do Sul

\begin{tabular}{|c|c|c|c|c|c|c|c|c|c|c|c|}
\hline Instituição & Cultura $^{(1)}$ & Solo & Argila & $\operatorname{MOS}^{(2)}$ & $\mathrm{pH} \mathrm{H}_{2} \mathrm{O}$ & $\mathbf{P}$ & $\mathbf{K}$ & $\mathrm{Ca}^{2+}$ & $\operatorname{Mg}^{2+}$ & $\mathrm{Al}^{3+}$ & CTC \\
\hline & & & \multicolumn{2}{|c|}{$-\mathrm{g} \mathrm{kg}^{-1} \_$} & & \multicolumn{2}{|c|}{ — $\mathrm{mg} \mathrm{kg}^{-1} \_$} & 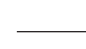 & \multicolumn{2}{|c|}{$\mathrm{cmol}_{\mathrm{c}} \mathrm{kg}^{-1}$} & \\
\hline EEA-UFRGS & M.T & PVd & 220 & 28 & 5,0 & 3,1 & 182 & 3,3 & 1,8 & 0,5 & 10,4 \\
\hline COTRISOJA-FUNDACEP & S.T.M & $\mathrm{LVd}$ & 480 & 31 & 5,3 & 12,0 & 113 & 6,0 & 2,8 & 0,3 & 13,9 \\
\hline COTRIJAL-FUNDACEP & S.T.M & $\mathrm{LVd}$ & 300 & 27 & 5,2 & 12,0 & 104 & 2,8 & 1,3 & 0,9 & 9,6 \\
\hline COTREL-FUNDACEP & S.T.M & LVaf & 700 & 37 & 5,2 & 9,4 & 248 & 8,6 & 4,2 & 0,4 & 18,7 \\
\hline COPALMA-FUNDACEP & S.M & $\mathrm{LVd}$ & 560 & 38 & 5,5 & 4,0 & 77 & 3,0 & 1,6 & 0,5 & 11,6 \\
\hline COTRIJUÍ-FUNDACEP & S.M & $\mathrm{LVd}$ & $>560$ & 47 & 5,9 & 18,0 & 63 & 5,1 & 2,1 & 0,0 & 9,9 \\
\hline COTRIPAL-FUNDACEP & S.M & $\mathrm{LVd}$ & $>560$ & 34 & 5,4 & 13,0 & 76 & 4,4 & 2,1 & 0,0 & 10,5 \\
\hline UFSM & S.M.P & PVd & 100 & - & 5,7 & 7,6 & 33 & 0,5 & 0,5 & 0,2 & 8,5 \\
\hline COTRISA-FUNDACEP & S.M.T & LVdf & 650 & 34 & 5,0 & 9,4 & 153 & 4,6 & 2,1 & 0,4 & 10,9 \\
\hline FUNDACEP & S.M.T & $\mathrm{LVd}$ & 500 & 38 & 5,4 & 28,0 & 88 & 6,6 & 3,2 & 0,2 & 14,3 \\
\hline COTRIJUÍ (Ajuricaba) & $\mathrm{S}$ & $\mathrm{LVd}$ & $>560$ & 36 & 6,2 & 5,8 & 151 & 7,4 & 3,9 & 0,0 & 14,5 \\
\hline COTRIJUÍ (C. Bicaco) & $\mathrm{S}$ & $\mathrm{LVd}$ & $>560$ & 38 & 6,5 & 17,0 & 203 & 7,9 & 4,2 & 0,0 & 15,7 \\
\hline COTRIJUÍ (Redentora) & $\mathrm{S}$ & $\mathrm{LVd}$ & $>560$ & 37 & 6,5 & 12,0 & 161 & 6,5 & 3,6 & 0,0 & 12,9 \\
\hline COTRIJUÍ (S. Augusto) & $\mathrm{S}$ & $\mathrm{LVd}$ & $>560$ & 35 & 6,2 & 7,7 & 237 & 7,6 & 4,0 & 0,0 & 14,6 \\
\hline COTRIJUÍ (S. Valério) & $\mathrm{S}$ & $\mathrm{LVd}$ & $>560$ & 36 & 5,3 & 11,0 & 149 & 4,2 & 2,2 & 0,4 & 11,6 \\
\hline COTRIJUÍ (S. Valério) & $\mathrm{S}$ & $\mathrm{LVd}$ & $>560$ & 38 & 6,1 & 5,6 & 44 & 7,7 & 2,9 & 0,0 & 14,1 \\
\hline COTRIBÁ (Ibirubá) & $\mathrm{S}$ & LVd & $>560$ & 38 & 5,6 & 8,3 & 65 & 5,4 & 2,7 & 0,0 & 12,2 \\
\hline
\end{tabular}

(1) Culturas; S: soja; T: trigo; M: milho; e P: pastagem. 
a lanço. No fator dose de $\mathrm{K}_{2} \mathrm{O}$, os tratamentos foram compostos pela testemunha com dose zero de $\mathrm{K}_{2} \mathrm{O}$, quantidades de $\mathrm{K}_{2} \mathrm{O}$ exportadas pelos grãos e 1 e 1,5 vezes a dose de $\mathrm{K}_{2} \mathrm{O}$ recomendada pela CFSRS/SC (1995). No fator cultura, os tratamentos foram compostos por uma parcela da sequência soja/trigo e três parcelas da sequência soja/aveia-preta, soja/aveiapreta + ervilhaça, milho/trigo (rotação de inverno e de verão em diferentes etapas, de modo a ter todas as culturas em todos os anos). Os experimentos foram realizados entre 1999 e 2001.

Um experimento foi conduzido pela Universidade Federal de Santa Maria, com doses de K (0, 50, 100 e $200 \mathrm{~kg} \mathrm{ha}{ }^{-1}$ de $\mathrm{K}_{2} \mathrm{O}$ ) aplicadas na superfície, na instalação do experimento em 1995, sob o sistema plantio direto de cultivo por quatro anos, com soja e milho no verão e aveia-preta no inverno. Os dados deste experimento foram publicados por Brunetto et al. (2005).

Os experimentos instalados em Santo Ângelo (COTRISA-FUNDACEP) e em Cruz Alta (FUNDACEP) foram adubados sempre a lanço, com doses de $\mathrm{K}(0$, $30,60,90$ e $120 \mathrm{~kg} \mathrm{ha}^{-1}$ de $\mathrm{K}_{2} \mathrm{O}$ ) nas parcelas principais aplicadas na cultura do trigo e doses de $\mathrm{K}$ recomendadas pela CFSRS/SC, 1995 (0, 1/3, 2/3, 3/3 e 4/3 da dose de $\mathrm{K}_{2} \mathrm{O}$ ) aplicadas nas subparcelas, nas culturas de soja e milho, sob o sistema plantio direto. Os experimentos foram realizados entre 1994 e 2001.

Os experimentos feitos pela COTRIJUI (seis locais) e pela COTRIBÁ (um local) com soja, em 2001/02, foram instalados em parcelas com doses de $\mathrm{K}(0,300$ e $600 \mathrm{~kg} \mathrm{ha}^{-1}$ de $\mathrm{K}_{2} \mathrm{O}$ ) aplicadas a lanço na superfície antes do cultivo da soja sob o sistema plantio direto.

As amostras de solo foram retiradas com pá-decorte, nas camadas de 0-20 e 0-10 cm de profundidade e 10/5 cm de largura/espessura. Os resultados de rendimento de grãos das culturas de soja, trigo e milho dos experimentos utilizados neste estudo foram obtidos a partir de colheitas realizadas pelas instituições que os instalaram.

As amostras de solo foram secas a $40-45^{\circ} \mathrm{C}$ em estufa com circulação forçada de ar, moídas em moinho de martelo e peneiradas ( $2 \mathrm{~mm})$. Nos tratamentostestemunha de cada experimento, com dose zero do fertilizante testado, o $\mathrm{K}$ foi determinado pelos métodos Mehlich-1 (Mehlich, 1953), resina em lâminas (Tedesco et al., 1995) e Mehlich-3 (Mehlich, 1984). Todos os procedimentos de laboratório foram feitos em duplicata, utilizando-se os dados médios na apresentação dos resultados.

As análises de regressão das curvas de produção de grãos das culturas em função das doses de K aplicadas no solo foram calculadas com os programas SIGMAPLOT e SIGMASTAT, utilizando-se equações de primeiro e de segundo grau que proporcionaram o melhor ajuste dos dados pelo método dos quadrados mínimos. Nas análises de regressão dos experimentos em que houve o cultivo de várias culturas com uma única aplicação de fertilizantes, foram utilizadas as mesmas doses em todos os cultivos. Nos experimentos que receberam mais de uma aplicação de fertilizantes, foi utilizado o somatório das doses aplicadas até a avaliação da cultura.

$\mathrm{O}$ rendimento relativo ( $\mathrm{RR}$, em \%) das culturas foi determinado a partir das funções de produção obtidas das suas respostas nos experimentos com doses de $\mathrm{K}$ (Equação 1). A calibração foi feita com os rendimentos relativos de cada experimento e o $\mathrm{K}$ determinado pelo método dos quadrados mínimos.

$$
\mathrm{RR}=\left(\mathrm{y}_{\mathrm{K} 0} / \mathrm{y}_{\max }\right) 100
$$

em que $\mathrm{y}_{\mathrm{k} 0}=$ rendimento com dose 0 de $\mathrm{K}$; $\mathrm{y}_{\max }=$ rendimento máximo.

A escolha da função utilizada no ajuste dos dados da curva de calibração foi feita pelo maior valor de $\mathrm{R}^{2}$ e selecionada pelo programa de computação "TABLE CURVE 2D v 5” da SPSS (1999). A equação selecionada $\left(\mathrm{y}=\mathrm{A}\left(1-10^{-\mathrm{bx}}\right)\right)$ foi a de Mitscherlich, que melhor se ajustou à maioria dos dados, passou próximo da origem e foi forçada a atingir $100 \%$ do rendimento relativo (Black, 1992; Brunetto et al., 2005; Wendling et al., 2008).

O teor crítico na calibração foi definido como o valor do nutriente no solo para a estimativa de $90 \%$ do rendimento relativo, como foi definido nos Programas de Adubação no Rio Grande do Sul e em Santa Catarina (Mielniczuk et al., 1969; UFRGS, 1973; 1976; FEGOTRIGO, 1981; Siqueira et al., 1987; CFSRS/ SC, 1995; 2004) e em São Paulo (Raij et al., 1997). As faixas de fertilidade foram ajustadas para teores de $\mathrm{K}$ determinados pelos métodos em valores inteiros e equidistantes, ou seja: teores muito baixos, baixos, médios, altos e muito altos.

\section{RESULTADOS E DISCUSSÃO}

Os coeficientes das equações de regressão, os coeficientes de determinação e os rendimentos relativos das culturas à adição de fertilizante potássico estão apresentados no quadro 2 e foram selecionados pelo maior coeficiente de determinação a partir dos rendimentos médios das diferentes culturas, locais e anos de cultivo.

Os rendimentos médios estimados de grãos de soja (2.345 kg ha-1), trigo (2.142 $\mathrm{kg} \mathrm{ha}^{-1}$ ) e milho (4.393 $\mathrm{kg} \mathrm{ha}^{-1}$ ), obtidos nos experimentos em parcelastestemunha do fertilizante potássico (coeficiente "a" da função de produção), foram um pouco superiores aos rendimentos médios do Rio Grande do Sul nas safras da década de 2000 (1.957, 1.746 e $3.140 \mathrm{~kg} \mathrm{ha}^{-1}$ para soja, trigo e milho, respectivamente-CONAB, 2009). Isso indica que, em muitos casos, os solos utilizados nos experimentos foram representativos do estado de fertilidade dos solos naquele Estado.

A soja foi a cultura que menos respondeu à aplicação do fertilizante e aumentou, em média, 4,30 kg de grãos 
Quadro 2. Instituição condutora dos experimentos no Rio Grande do Sul, ano de condução, equação de regressão de produção de grãos, coeficiente de determinação $\left(R^{2}\right)$ e rendimento relativo das culturas soja trigo e milho ( $\mathrm{kg} \mathrm{ha}^{-1}$ ) cultivadas sob o sistema plantio direto, em função das doses de $\mathrm{K}_{2} \mathrm{O}\left(\mathrm{kg} \mathrm{ha}^{-1}\right)$

\begin{tabular}{|c|c|c|c|c|}
\hline Instituição & Ano & Equação de regressão & $\mathbf{R}^{2}$ & Rendimento relativo \\
\hline & & Soja & & $\%$ \\
\hline COTRIJUI (Ajuricaba) & 2001 & $\hat{y}=3.821-0,49 x$ & 0,98 & 100 \\
\hline COTRIJUI (C,Bicaco) & 2001 & $\hat{\mathrm{y}}=2.761+0,38 \mathrm{x}$ & 0,23 & 93 \\
\hline COTRIJUI (Redentora) & 2001 & $\hat{\mathrm{y}}=2.144+0,37 \mathrm{x}$ & 0,11 & 91 \\
\hline COTRIJUI (S, Augusto) & 2001 & $\hat{\mathrm{y}}=1.834+0,68 \mathrm{x}$ & 0,97 & 82 \\
\hline COTRIBÁ (Ibirubá) & 2001 & $\hat{y}=3.464-0,03 x$ & 0,11 & 100 \\
\hline COTRIJUI (S, Valério) & 2001 & $\hat{\mathrm{y}}=2.987+1,23 \mathrm{x}$ & 0,99 & 87 \\
\hline COTRIJUI (S, Valério) & 2001 & $\hat{\mathrm{y}}=2.731-2,46 \mathrm{x}$ & 0,82 & 100 \\
\hline COTRISOL - FUNDACEP & 1999 & $\hat{y}=3.662+10,39 x-0,090 x^{2}$ & 0,98 & 92 \\
\hline COTRISOL - FUNDACEP & 2000 & $\hat{y}=2.585+7,08 x-0,020 x^{2}$ & 0,99 & 83 \\
\hline COTRISOL - FUNDACEP & 2001 & $\hat{\mathrm{y}}=1.403+7,08 \mathrm{x}-0,020 \mathrm{x}^{2}$ & 0,96 & 73 \\
\hline COTRIJAL-FUNDACEP & 1999 & $\hat{y}=1.932+11,13 x-0,050 x^{2}$ & 0,94 & 82 \\
\hline COTRIJAL -FUNDACEP & 2000 & $\hat{\mathrm{y}}=2.886+6,30 \mathrm{x}$ & 0,75 & 82 \\
\hline COTRIJAL - FUNDACEP & 2001 & $\hat{\mathrm{y}}=1.837+7,61 \mathrm{x}-0,040 \mathrm{x}^{2}$ & 0,89 & 84 \\
\hline COTREL - FUNDACEP & 1999 & $\hat{\mathrm{y}}=1.882+7,00 \mathrm{x}-0,060 \mathrm{x}^{2}$ & 0,79 & 90 \\
\hline COPALMA-FUNDACEP & 1999 & $\hat{\mathrm{y}}=322+1,94 \mathrm{x}-0,002 \mathrm{x}^{2}$ & 0,99 & 61 \\
\hline UFSM (S, Fransisco) & 1995 & $\hat{y}=2.606+3,86 x-0,010 x^{2}$ & 0,60 & 88 \\
\hline UFSM (S, Fransisco) & 1996 & $\hat{\mathrm{y}}=2.837+6,28 \mathrm{x}-0,020 \mathrm{x}^{2}$ & 0,61 & 85 \\
\hline UFSM (S, Fransisco) & 1997 & $\hat{\mathrm{y}}=2.303+5,31 \mathrm{x}-0,010 \mathrm{x}^{2}$ & 0,60 & 82 \\
\hline UFSM (S, Fransisco) & 1998 & $\hat{y}=1.186+2,69 x-0,008 x^{2}$ & 0,87 & 83 \\
\hline COTRIJU-FUNDACE P & 2001 & $\hat{y}=2.335+7,00 x-0,004 x^{2}$ & 0,99 & 82 \\
\hline COTRIPAL-FUNDACEP & 2001 & $\hat{y}=1.526+7,89 x-0,006 x^{2}$ & 0,91 & 67 \\
\hline COTRISA-FUNDACEP & 1995 & $\hat{y}=2.372+2,96 x-0,008 x^{2}$ & 0,47 & 90 \\
\hline COTRISA-FUNDACEP & 1996 & $\hat{\mathrm{y}}=2.108+3,34 \mathrm{x}-0,004 \mathrm{x}^{2}$ & 0,27 & 84 \\
\hline COTRISA-FUNDACEP & 1999 & $\hat{\mathrm{y}}=1.586+4,47 \mathrm{x}-0,010 \mathrm{x}^{2}$ & 0,44 & 82 \\
\hline COTRISA-FUNDACEP & 2000 & $\hat{\mathrm{y}}=1.887+2,35 \mathrm{x}-0,005 \mathrm{x}^{2}$ & 0,05 & 91 \\
\hline FUNDACEP & 1994 & $\hat{\mathrm{y}}=2.784+6,31 \mathrm{x}-0,010 \mathrm{x}^{2}$ & 0,42 & 84 \\
\hline FUNDACEP & 1997 & $\hat{y}=3.348-2,08 x$ & 0,07 & 100 \\
\hline FUNDACEP & 2000 & $\hat{\mathrm{y}}=2.536+12,40 \mathrm{x}-0,080 \mathrm{x}^{2}$ & 0,39 & 84 \\
\hline \multirow[t]{2}{*}{ Média } & & $\hat{\mathrm{y}}=2.345+4,32 \mathrm{x}-0,020 \mathrm{x}^{2}$ & 0,75 & 75 \\
\hline & & Trigo & & \\
\hline COTRISOL -FUNDACEP & 2000 & $\hat{\mathrm{y}}=2.591+57,10 \mathrm{x}-0,815 \mathrm{x}^{2}$ & 0,99 & 80 \\
\hline COTRIPAL - FUNDACEP & 2000 & $\hat{\mathrm{y}}=1.176+25,73 \mathrm{x}-0,340 \mathrm{x}^{2}$ & 0,96 & 71 \\
\hline COTREL-FUNDACEP & 2000 & $\hat{\mathrm{y}}=1.632+10,16 \mathrm{x}-0,040 \mathrm{x}^{2}$ & 0,66 & 74 \\
\hline COTRISA -FUNDACEP & 1996 & $\hat{y}=2.723+2,70 x-0,010 x^{2}$ & 0,02 & 96 \\
\hline COTRISA - FUNDACEP & 1999 & $\hat{\mathrm{y}}=1.809+3,27 \mathrm{x}-0,008 \mathrm{x}^{2}$ & 0,39 & 87 \\
\hline FUNDACEP & 1997 & $\hat{\mathrm{y}}=2.206+5,15 \mathrm{x}-0,030 \mathrm{x}^{2}$ & 0,15 & 91 \\
\hline FUNDACEP & 1999 & $\hat{\mathrm{y}}=2.860+4,96 \mathrm{x}-0,060 \mathrm{x}^{2}$ & 0,23 & 97 \\
\hline \multirow[t]{2}{*}{ Média } & & $\hat{y}=2.142+15,58 x-0,168 x^{2}$ & 0,49 & 85 \\
\hline & & Milho & & \\
\hline UFRGS & 2000 & $\hat{y}=9.212+2,85 x-0,020 x^{2}$ & 0,01 & 90 \\
\hline COTRISOL - FUNDACEP & 1999 & $\hat{y}=2.582+63,32 x-0,250 x^{2}$ & 0,79 & 61 \\
\hline COTRISOL - FUNDACEP & 2000 & $\hat{\mathrm{y}}=4.215+27,79 \mathrm{x}-0,020 \mathrm{x}^{2}$ & 0,99 & 87 \\
\hline COTRISOL - FUNDACEP & 2001 & $\hat{y}=3.576+23,27 x-0,120 x^{2}$ & 0,83 & 77 \\
\hline COTRIJAL - FUNDACEP & 1999 & $\hat{y}=3.403+100,49 x-2,910 x^{2}$ & 0,99 & 80 \\
\hline COTRIJAL - FUNDACEP & 2000 & $\hat{\mathrm{y}}=3.958+13,84 \mathrm{x}$ & 0,63 & 76 \\
\hline COTRIJAL - FUNDACEP & 2001 & $\hat{\mathrm{y}}=2.860+13,39 \mathrm{x}$ & 0,88 & 63 \\
\hline COTREL-FUNDACEP & 1999 & $\hat{y}=7.010+204,85 x-4,170 x^{2}$ & 0,94 & 74 \\
\hline COPALMA - FUNDACEP & 1999 & $\hat{y}=1.197+10,56 x-0,030 x^{2}$ & 0,80 & 59 \\
\hline UFSM (S, Francisco) & 1999 & $\hat{\mathrm{y}}=3.629+19,91 \mathrm{x}-0,040 \mathrm{x}^{2}$ & 0,97 & 61 \\
\hline COTRIJUI - FUNDACEP & 2001 & $\hat{\mathrm{y}}=3.767+15,54 \mathrm{x}-0,100 \mathrm{x}^{2}$ & 0,83 & 83 \\
\hline COTRIPAL - FUNDACEP & 2001 & $\hat{\mathrm{y}}=3.903+20,00 \mathrm{x}-0,080 \mathrm{x}^{2}$ & 0,99 & 69 \\
\hline COTRISA-FUNDACEP & 1994 & $\hat{\mathrm{y}}=7.801+6,93 \mathrm{x}-0,020 \mathrm{x}^{2}$ & 0,06 & 94 \\
\hline Média & & $\hat{y}=4.393+40,21 x-0,0705 x^{2}$ & 0,75 & 75 \\
\hline
\end{tabular}

para cada $\mathrm{kg}$ de $\mathrm{K}_{2} \mathrm{O}$ adicionado ao solo. $\mathrm{O}$ trigo teve respostas intermediárias e aumentou em média $15,58 \mathrm{~kg}$ de grãos para cada $\mathrm{kg}$ de $\mathrm{K}_{2} \mathrm{O}$ adicionado ao solo. O milho teve o maior aumento, em média 40,21 kg de grãos para cada kg de $\mathrm{K}_{2} \mathrm{O}$ adicionado ao solo (Quadro 2). Menores rendimentos por unidade de $\mathrm{K}_{2} \mathrm{O}$ aplicado na soja em relação ao rendimento de trigo e milho também foram encontrados por Wendling et al. (2008). 
A soja necessita de mais $\mathrm{K}_{2} \mathrm{O}$ para a produção de 1 t de grãos, se comparada com o trigo e milho. Esses dados estão de acordo com os de Raij et al. (1997), que citaram que as plantas inteiras precisam de $\mathrm{K}_{2} \mathrm{O}$ na quantidade de 60 (estimado pelos teores em tecidos), 27,7 e $21,7 \mathrm{~kg} \mathrm{t}^{-1}$ de grãos de soja, trigo e milho, respectivamente. Supondo-se uma produção de $90 \%$ da máxima eficiência técnica, o que corresponde, em termos práticos, à máxima eficiência econômica, o rendimento de grãos de soja, trigo e milho, baseado no quadro 2 , seria de 2,$6 ; 2,2$; e $5,7 \mathrm{t} \mathrm{ha}^{-1}$ e a necessidade de $\mathrm{K}$ na forma de $\mathrm{K}_{2} \mathrm{O}$, de 156,61 e $124 \mathrm{~kg} \mathrm{ha}^{-1}$, respectivamente.

A quantidade exportada de $\mathrm{K}_{2} \mathrm{O}$ pelos grãos também foi maior na cultura da soja, se comparada às quantidades para o trigo e para o milho (Raij et al., 1997; CFSRS/SC, 2004). Supondo-se rendimento de $90 \%$ da máxima eficiência técnica, que seria de 2,6 ; 2,2 ; e $5,7 \mathrm{t} \mathrm{ha}^{-1}$, e na exportação de $\mathrm{K}$ segundo a CFSRS/SC (2004), que seria de 20; 6 e $6 \mathrm{~kg} \mathrm{t}^{-1} \mathrm{de} \mathrm{K}_{2} \mathrm{O}$, a exportação seria de $52 ; 13$ e $34 \mathrm{~kg} \mathrm{ha}^{-1}$ de $\mathrm{K}_{2} \mathrm{O}$ de soja, trigo e milho, respectivamente.
A eficiência agronômica dos fertilizantes foi, portanto, maior para o milho e menor para a soja. No entanto, ao implantar uma lavoura no Rio Grande do Sul, considerou-se o aspecto econômico e, nesse caso, a cultura da soja foi mais eficiente devido ao valor de mercado do grão produzido, ao baixo custo de implantação e condução da lavoura e ao menor risco de frustração de safra, se comparada às culturas do trigo e do milho (FECOAGRO/RS, 2004).

Os coeficientes de determinação (Quadro 2) das equações do rendimento em função de doses do nutriente em estudo foram, em média, altos na maioria dos resultados, mostrando que houve ajuste satisfatório.

As curvas de calibração, as funções e os teores críticos de K (Figura 1) foram determinados pelos métodos Mehlich-1, Mehlich-3 e resina de troca iônica, em amostras de solo das camadas de 0-20 e 0-10 cm de profundidade. Os teores críticos foram estimados pelos teores de $\mathrm{K}$ do solo, sem a adição do nutriente testado com o rendimento relativo de $90 \%$, a partir dos resultados do quadro 1.
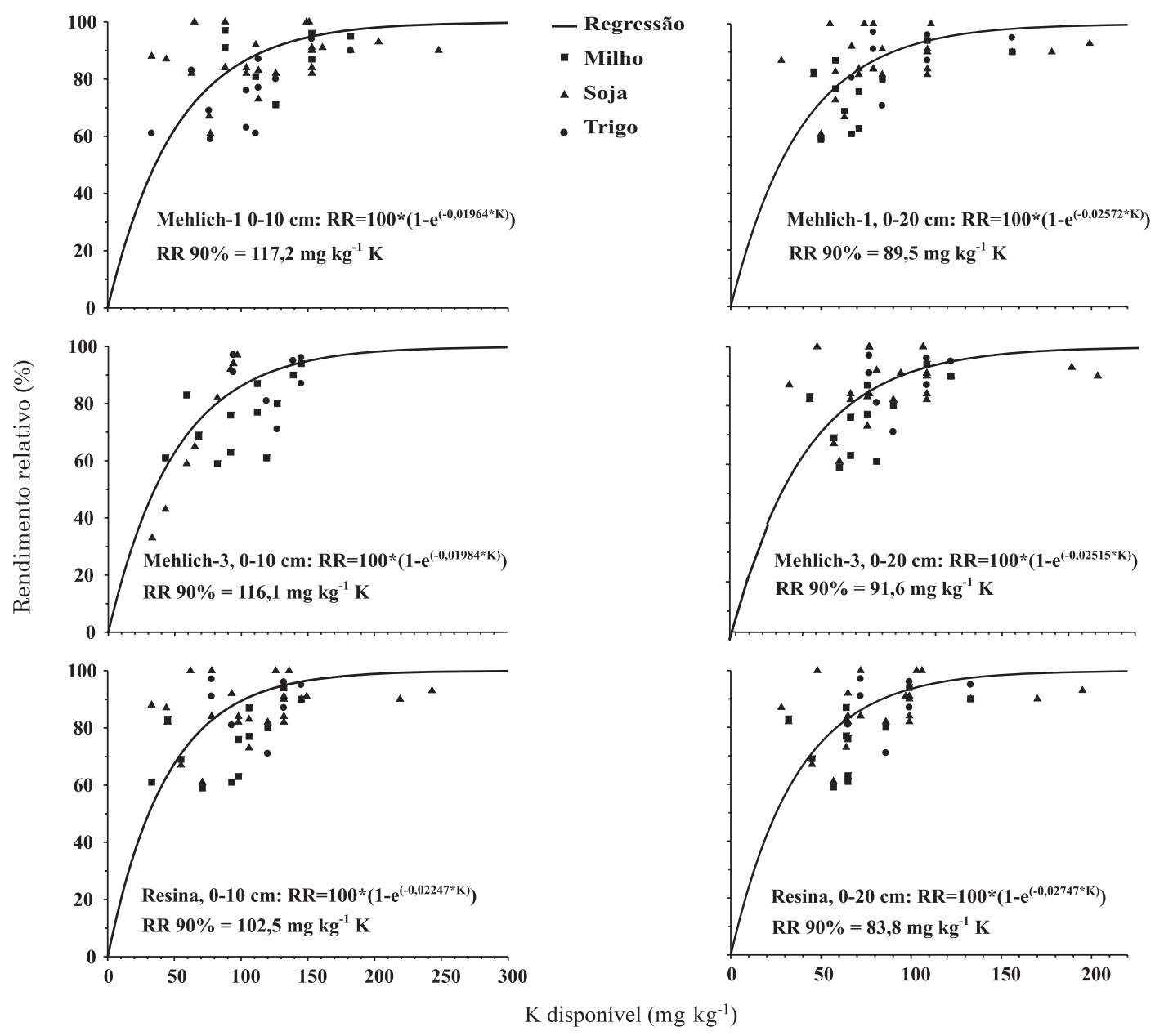

Figura 1. Rendimento relativo de milho, soja e trigo cultivados em sistema plantio direto e teores de $\mathrm{K}$ em solos amostrados nas camadas de 0-10 e 0-20 cm de profundidade, determinado pelos métodos Mehlich1, Mehlich-3 e resina. 
Os níveis críticos para o K obtidos pelos métodos de análise de solo Mehlich-1 e Mehlich-3 apresentaram valores próximos, e ambos tiveram níveis críticos um pouco superiores ao obtido pelo método da resina. Essa tendência foi semelhante nas amostras das duas camadas amostradas (Figura 1). Os teores críticos muito próximos entre os métodos Mehlich-1 e Mehlich3 , que são extratores ácidos, estão associados à capacidade deles em extrair o K disponível que se correlaciona significativamente com a resposta de plantas (Oliveira, 1970; Mielniczuk \& Selbach, 1978; Nachtigall \& Vahl, 1991; Kroth, 1998; Schlindwein, 2003). O método Mehlich-1 foi utilizado nas análises de rotina nos laboratórios gaúchos e catarinenses, já o método Mehlich-3 foi considerado promissor, pela praticidade em laboratório e boa correlação com respostas de plantas e por extrai simultaneamente $P$, K, Ca, Mg, Na, Mn, Cu e Zn (Mehlich, 1984), o que pode resultar em maior eficiência nos laboratórios. O método da resina atualmente é utilizado em análises de $\mathrm{P}$ em rotina nos citados laboratórios e extrai o $\mathrm{K}$ por troca iônica. Os menores teores críticos de K determinados pelo método da resina estão associados aos mecanismos da troca e possível competição dos sítios de troca por outros cátions básicos, como Ca e Mg presentes em maior proporção em alguns solos de maior CTC (Kroth, 1998).

A diferença entre teores críticos obtidos neste trabalho e os obtidos em calibrações anteriores está na grandeza numérica. $\mathrm{O}$ teor obtido pelo método Mehlich-1 na camada de $0-20 \mathrm{~cm}\left(89,5 \mathrm{mg} \mathrm{kg}^{-1}\right.$ de solo) foi $50 \%$ maior do que o valor de $60 \mathrm{mg} \mathrm{dm}^{-3}$ obtido em calibrações anteriores feitas no Rio Grande do Sul e em Santa Catarina e utilizado como teor crítico até 1986 (Mielniczuk et al., 1969; UFRGS, 1973; 1976; FEGOTRIGO, 1981) e maior do que o valor de $80 \mathrm{mg} \mathrm{dm}^{-3}$, utilizado a partir das recomendações de Siqueira et al. (1987) e da CFSRS/SC (1995; 2004).

O teor crítico de $\mathrm{K}$ na camada de $0-10 \mathrm{~cm}$ de profundidade foi em torno de $30 \%$ maior do que na camada de 0-20 cm. Assim como o P, o K também se acumula na superfície e subsuperfície do solo sob o sistema plantio direto (Eltz et al., 1989; Schlindwein \& Anghinoni, 2000). No trabalho de Schlindwein \& Anghinoni (2000) realizado em experimentos de longa duração sob esse sistema (experimentos com 10, 12, 12 e 19 anos de condução), o aumento de K na camada de $0-10 \mathrm{~cm}$ em relação à de $0-20 \mathrm{~cm}$ de profundidade também foi em torno de $30 \%$. Segundo Giacomini et al. (2003), o acúmulo de $\mathrm{K}$ é favorecido, pois aproximadamente $75 \%$ desse nutriente absorvido pelas plantas e remanescente nos resíduos retorna ao solo após 30 dias, o que foi verificado em diversas espécies de plantas de coberturas cultivadas em sistema plantio direto.

Em 2004, a CFSRS/SC (2004) adotou como nível crítico de $\mathrm{K}$ os valores de 45, 60 e $90 \mathrm{mg} \mathrm{dm}^{-3}$ para os solos com CTC < 5; 5,1-15 e > $15 \mathrm{cmol}_{\mathrm{c}} \mathrm{dm}^{-3}$, respectivamente. Neste estudo, das 48 avaliações, duas foram feitas em dois solos com $\mathrm{CTC}_{(\mathrm{pH} 7,0)}$ $>15 \mathrm{cmol}_{\mathrm{c}} \mathrm{dm}^{-3}$, os quais teriam nível crítico em torno de $90 \mathrm{mg} \mathrm{kg}^{-1}$ de $\mathrm{K}$ na camada de $0-20 \mathrm{~cm}$ de profundidade, semelhante ao estabelecido pela CFSRS/ SC (2004). Entretanto, a maioria das avaliações de rendimento de grãos (46) ocorreu em 15 solos que apresentavam $\mathrm{CTC}_{(\mathrm{pH} 7,0)}$ entre 5,1-15 $\mathrm{cmol}_{\mathrm{c}} \mathrm{kg}^{-1}$, portanto com nível crítico $50 \%$ maior do que o definido pela CFSRS/SC (2004).

Os resultados que deram origem às curvas de calibração (Figura 1) foram os mesmos utilizados por Schlindwein (2003), que utilizou outra função na curva de calibração $(\hat{y}=a+b \operatorname{lnx})$ e encontrou nível crítico ajustado de 120 e de $180 \mathrm{mg} \mathrm{kg}^{-1} \mathrm{de} \mathrm{K}$, amostrado nas camadas de $0-10$ e de $0-20 \mathrm{~cm}$, respectivamente. Neste trabalho, a curva de calibração foi feita com a equação de Mitcherlich, tal como nos trabalhos de Brunetto et al. (2005), que encontraram nível crítico de $\mathrm{K}$ de $42 \mathrm{mg} \mathrm{dm}^{-3}$, e de Wendling et al. (2008), que obtiveram nível crítico de $\mathrm{K}$ de $75 \mathrm{mg} \mathrm{dm}^{-3}$, sendo em ambos os trabalhos os solos amostrados na camada de $0-10 \mathrm{~cm}$ de profundidade.

O nível crítico de 42 e de $75 \mathrm{mg} \mathrm{dm}^{-3}$ foram em torno de $1 / 3$ e $3 / 4$, respectivamente, menores do que os níveis críticos na camada de $0-10 \mathrm{~cm}$ de profundidade (Figura 1). O trabalho de Brunetto et al. (2005) foi feito em dois Argissolos e o de Wendling et al. (2008), em três Argissolos e quatro Latossolos do Paraguai. Neste trabalho, o estudo de calibração foi feito em 15 Latossolos e dois Argissolos. Normalmente, solos arenosos têm menor CTC e menor nível crítico, como nos programas de recomendação do Rio Grande do Sul e de Santa Catarina (CFSRS/ SC, 2004), no Cerrado (Sousa \& Lobato, 2002).

Nível crítico menor de K (40 $\left.\mathrm{mg} \mathrm{kg}^{-1}\right)$ também foi encontrado por Borkert et al. (1993) estudando, por vários anos, em um único Latossolo do Paraná e por Scherer (1998), que constatou nível crítico de K de $63 \mathrm{mg} \mathrm{dm}^{-3}$ estudando, por vários anos, em um único Latossolo de Santa Catarina, ambos amostrados na camada de $0-20 \mathrm{~cm}$ de profundidade do solo cultivado no sistema convencional.

Após a definição do nível crítico de K por diferentes métodos de análise e profundidade de amostragem, pode-se detalhar melhor a função na porção da curva abaixo desse ponto, estabelecendo-se faixas de teores. Essas faixas podem ser divididas a partir do rendimento relativo, como foi estabelecido nas Recomendações de Adubação e Calagem para o Estado de São Paulo (Raij et al., 1997), ou em teores no solo em quantidades equidistantes, tal como estabelecido no Rio Grande do Sul (Mielniczuk et al., 1969; UFRGS, 1973; 1976; FEGOTRIGO, 1981; Manual..., 1981; Siqueira et al., 1987; CFSRS/SC, 1995; 2004). Neste trabalho, optou-se por estabelecer faixas de fertilidade do solo com valores ajustados para números inteiros e, ou, que facilitassem a divisão em faixas equidistantes, denominados muito baixos, baixos, médios, altos e muito altos (Quadro 2). 
Quadro 3. Faixas ajustadas de fertilidade de K do solo cultivado no sistema plantio direto, determinado em diferentes profundidades e por diferentes métodos

\begin{tabular}{|c|c|c|c|c|c|c|}
\hline \multirow{2}{*}{$\begin{array}{l}\text { Profundidade } \\
\text { de amostragem }\end{array}$} & \multirow{2}{*}{ Extrator } & \multicolumn{5}{|c|}{ Classe de fertilidade } \\
\hline & & Muito alta & Baixa & Adequador $^{(1)}$ & Alta & Muito alta \\
\hline \multicolumn{2}{|l|}{$\mathrm{cm}$} & & & \multicolumn{2}{|l|}{$-\mathrm{mg} \mathrm{kg}^{-1}$} & \\
\hline \multirow[t]{3}{*}{$0-20$} & Mehlich-1 & $0-30$ & $31-60$ & $61-90$ & $91-120$ & $>120$ \\
\hline & Mehlich-3 & $0-30$ & $31-60$ & $61-90$ & $91-120$ & $>120$ \\
\hline & Resin & $0-28$ & $29-56$ & $57-84$ & $85-112$ & $>112$ \\
\hline \multirow[t]{3}{*}{$0-10$} & Mehlich-1 & $0-40$ & $41-80$ & $81-120$ & $121-160$ & $>160$ \\
\hline & Mehlich-3 & $0-40$ & $41-80$ & $81-120$ & $121-160$ & $>160$ \\
\hline & Resin & $0-34$ & $35-68$ & $69-102$ & $103-137$ & $>137$ \\
\hline
\end{tabular}

(1) Valor superior da faixa corresponde ao teor crítico ajustado.

Ao ajustar as faixas de fertilidade do solo, determinaram-se novos níveis críticos para facilitar as interpretações de fertilidade em faixas de teores e para estabelecer as doses de fertilizantes com o objetivo de máxima eficiência econômica e aumento gradativo da fertilidade. Assim, o valor superior da faixa de teor médio representa o teor crítico ajustado (Quadro 3).

\section{CONCLUSÕES}

1. A resposta das plantas à adubação com $\mathrm{K}$ é maior na sequência milho, trigo, soja.

2. Os teores críticos de K determinados por Mehlich1 e Mehlich-3 foram semelhantes e um pouco superiores aos encontrados pelo método da resina nas camadas de 0-20 e 0-10 cm de profundidade.

3. O nível crítico de $\mathrm{K}$ em solos sob o sistema plantio direto de cultivo de soja, trigo e milho foi maior do que no atual programa de recomendações calibrado no sistema convencional de cultivo, tanto para solos amostrados na camada de 0-20 quanto na camada de 0-10 $\mathrm{cm}$ de profundidade.

\section{AGRADECIMENTOS}

Às instituições de ensino, pesquisa e extensão do Rio Grande do Sul, por gentilmente terem cedido amostras de solos de seus experimentos e os dados de rendimento de grãos.

\section{LITERATURA CITADA}

ANGHINONI, I. \& VOLKWEIS, J.S. Recomendações de uso de fertilizantes. In: SIMPÓSIO SOBRE FERTILIZANTES NA AGRICULTURA BRASILEIRA, 1984, Brasília. Anais.. Brasília, Embrapa/DEP, 1984. p.179-204.
BLACK, C.A. Soil fertility evaluation and control. Soil testin and fertilizer requeriment. Boca Raton, Lewis Pulishers, 1992. p.271-452.

BORKERT, C.M.; SFREDO, G.J. \& SILVA, D.N. Calibração de potássio trocável para a soja em um Latossolo Roxo distrófico. R. Bras. Ci. Solo, 17:223-226, 1993.

BRUNETTO, G.; GATIBONI, L.C.; RHEINHEIMER, D.S.; SAGIM, A. \& KAMINSKI, J. Nível crítico e respostas das culturas ao potáassio em um Argissolo sob sistema plantio direto. R. Bras. Ci. Solo, 29:565-571, 2005.

CATE, R.B. \& NELSON, L.A. Discontinuous models for rapid correlation, interpretation and utilization of soil analysis and fertilizer response data. International soil fertility evaluation and improvement program [S.l.], North Carolina, 1973. 77p. (Technical Bulletin, 7)

COMISSÃO DE FERTILIDADE DO SOLO - CFSRS/SC. Manual de adubação e de calagem para os estados do Rio Grande do Sul e Santa Catarina. 10.ed. Porto Alegre, SBCS - Núcleo Regional Sul/UFRGS, 2004. 394p.

COMISSÃO DE FERTILIDADE DO SOLO - CFSRS/SC. Recomendações de adubação e calagem para os estados do Rio Grande do Sul e Santa Catarina. 3.ed. Passo Fundo, SBCS - Núcleo Regional Sul: Embrapa/CNPT, 1995. 224p.

COMISSÃO DE FERTILIDADE DO SOLO DO ESTADO DE MG - CFSEMG. Recomendação para o uso de corretivos e fertilizantes em Minas Gerais. 5 aproximação. Viçosa, MG, 1999. 359p.

COMPANHIA NACIONAL DE ABASTECIMENTO - CONAB. Central de informações agropecuárias - safras - grãos. Disponível em: <http://www.conab.gov.br > Acesso em 08 de abril de 2009.

ELTZ, F.L.P.; PEIXOTO, R.T.G. \& JASTER F. Efeitos de sistemas de preparo do solo nas propriedades físicas e químicas de um Latossolo Bruno álico. R. Bras. Ci. Solo, 13:259-267, 1989.

EMATER/RS. Área plantada e rendimento das principais culturas. Porto Alegre, Emater/RS-DEPLAN/DIDOC, 1998 (Tabelas não publicadas) 
EVANS, E.E. Soil test calibration. In: BROWN, J.R., ed. Soil testing: Sampling, correlation, calibration end interpretation. Madison, Soil Science Society of America, 1987. p.23-29 (Special Publication, 21)

FECOTRIGO. Manual de adubação e calagem para cultivos agrícolas do Rio Grande do Sul e Santa Catarina. Porto Alegre, 1981. 34p. (Trigo e Soja, 56)

FEDERAÇÃO DAS COOPERATIVAS AGROPECUÁRIAS DO RIO GRANDE DO SUL - FECOAGRO/RS. Custo de produção, lavouras em plantio direto. Porto Alegre, 2004. $35 \mathrm{p}$.

KROTH, P.L. Disponibilidade de fósforo no solo para as plantas e fatores que afetam a extração por resina de troca em membranas. Porto Alegre, Universidade Federal do Rio Grande do Sul, 1998. 167p. (Tese de Mestrado)

MEHLICH, A. Determination of $\mathrm{P}, \mathrm{Ca}, \mathrm{Mg}, \mathrm{K}, \mathrm{Na}$ and $\mathrm{NH} 4$ by North Carolina Soil Testing Laboratories. Raleigh, University of North Carolina, 1953.

MEHLICH, A. Mehlich 3 soil test extractant: A modification of Mehlich 2 extractant. Comm. Soil Sci. Plant Anal., 15:14091416, 1984.

MIELNiCZUK, J. \& SELBACH, P.A. Capacidade de suprimento de potássio de seis solos do Rio Grande do Sul. R. Bras. Ci. Solo, v.2, p.115-120, 1978.

MIELNICZUK, J.; LUDWICK, A. \& BOHNEN, H. Recomendações de adubo e calcário para as principais culturas do Estado do Rio Grande do Sul. Porto Alegre, Universidade Federal do Rio Grande do Sul, 1969. 36p. (Boletim Técnico, 2)

NACHTIGALL, G.R. \& VAHL, L.C. Capacidade de suprimento de potássio dos solos da região sul do Rio Grande do Sul. R. Bras. Ci. Solo, v.15, p.37-42, 1991.

OLIVEIRA, V. Formas de potássio em 21 solos do Rio Grande do Sul e sua capacidade de suprir potássio às plantas. Porto Alegre, Universidade Federal do Rio Grande do Sul, 1970. 91p. (Tese de Mestrado)

RAIJ, B.van; CANTARELLA, H.; QUAGGIO, J.A. \& FURLANI, A.M.C. Recomendações de adubação e calagem para o estado de São Paulo. 2.ed. Campinas, Instituto Agronômico/Fundação IAC, 1997. 285p. (Boletim Técnico, 100)

RAIJ, B.van; QUAGGIO, J.A. \& SILVA, M.N. Extraction of phosphorus, potassium, calcium and magnesium from soil by an ion-exchange resin procedure Comm. Soil Sci. Plant Anal., 17:547-566, 1986.
ROUSE, R.D. Soil test theory and calibration for cotton, corn, soybean and coastal Bermuda grass. Auburn, Agricultural Experiment Station Auburn University, 1968. 65p. (Technical Bulletin, 375)

SCHERER, E.E. Resposta da soja à adubaçãopotássica em um Latossolo húmico distrófico num período de doze anos. R. Bras. Ci. Solo, 22:49-55, 1998.

SCHLINDWEIN, J.A. Calibração de métodos de determinação de fósforo e potássiso do solo sob sistema plantio direto. Porto Alegre, Universidade Federal do Rio Grande do Sul, 2003. 169p. (Tese de Doutorado)

SCHLINDWEIN, J.A. \& ANGHINONI, I. Variabilidade vertical de fósforo e potássio disponíveis e profundidade de amostragem do solo no sistema plantio direto. Ci. Rural, 30:611-617, 2000.

SIQUEIRA, O. J. F.; SCHERER, E. E.; TASSINARI, G.; AGHINONI, I.; PATELLA, I. F.; TEDESCO. M. J.; MILAN, P. A. \& ERNANI, P. R. Recomendações de adubação e calagem para os estados do Rio Grande do Sul e Santa Catarina. Passo Fundo, Embrapa/CNPT, 1987. 100p.

SLATON, N.A.; GOLDEN, B.R.; NORMAN, R.J.; WILSON, C.E. \& DELONG, R.E. Correlation and calibration of soil potassium availability with rice and nutritional status. Soil Sci. Soc. Am. J., 73:1192-1201, 2009.

SOUSA, D.M.G.S. \& LOBATO, E. Cerrado correção do solo e adubação. Planaltina, Embrapa Cerrado, 2002. 416p.

TEDESCO, M.J.; GIANELLO, C. \& BISSANI, C.A. Análise de solo, plantas e outros materiais. 2.ed. Porto Alegre: Universidade Federal do Rio Grande do Sul, 1995. 147p. (Boletim Técnico, 5)

UNIVERSIDADE FEDERAL DO RIO GRANDE DO SUL UFRGS. Tabelas de adubação corretiva e adubação de manutenção para solos e culturas dos estados do Rio Grande do Sul e Santa Catarina. Trigo e Soja, v.10, p.1523, 1976.

UNIVERSIDADE FEDERAL DO RIO GRANDE DO SUL UFRGS. Tabelas de adubação corretiva e adubação de manutenção para os solos e culturas dos estados do Rio Grande do Sul e Santa Catarina. Porto Alegre, 1973. 11p. (Boletim Técnico)

WENDLING, A.; ELTZ, F.L.F.; CUBILLA, M.M.; AMADO, T.J.C. \& MIELNICZUK, J. Recomendação de adubação potássica para trigo, milho e soja sob sistema plantio direto no Paraguai. R. Bras. Ci. Solo, 32:1929-1939, 2008. 
